\title{
Impact of the Ayurveda Treatment Protocol in the Management of COVID -19
}

\author{
Sailekha P. ${ }^{1}$, Sudhikumar K B ${ }^{2}$ \\ ${ }^{1}$ Research Assistant, School of Fundamental Research in Ayurveda, Tripunithura, Kerala \\ ${ }^{2}$ Professor, School of Fundamental Research in Ayurveda, Tripunithura, Kerala \\ Corresponding Author: Sailekha P.
}

DOI: https://doi.org/10.52403/ijhsr.20220106

\begin{abstract}
Covid-19 is an infectious disease caused by SARS-COV-2, which can cause severe respiratory illness and complications in patients. Recognizing Ayurveda's endless possibilities for improving immunity and preventing disease progression, the Ministry of AYUSH developed and approved the Ayurvedic protocol for handling Covid-19. Even though Ayurveda practitioners have been prescribing medicines to the Covid-19 patients since approval, no studies have been reported from Kerala regarding the effectiveness of the Ayurveda Treatment approaches. Hence the School of Fundamental Research in Ayurveda designed an observational study to evaluate the impact of the Ayurveda Treatment approaches in the management of Covid -19. The study result affirms that the Ayurvedic treatment method is very effective against Covid-19 and the role of Ayurveda in addressing the Covid-19 challenge is invaluable.
\end{abstract}

Keywords: Covid-19, Ayurvedic protocol, School of Fundamental Research in Ayurveda

\section{INTRODUCTION}

Covid-19 is an infectious disease caused by SARS-COV-2, which can cause severe respiratory illness and complications in patients ${ }^{[1]}$. Around the world, it is hard work to find effective antiviral agents, immunomodulatory agents, and vaccines. But no effective solution has yet been found. As the epidemic continues to grow globally, health care systems are inadequate, even in developed countries. While supportive hospital care for critically ill patients can help with recovery, the increase in the number of patients and the shortage of beds is a challenge to get timely care and attention. Patients with mild to moderate symptoms are seen isolated at home.

In India, the spread of Covid-19 was relatively slow; However, due to the easing of lockdown restrictions, the country is experiencing a terrible jump in Covid-19 cases. In many cities, hospitals are unable to respond to this challenging situation.

India is a country where modern medical systems co-exist with indigenous medical systems such as Ayurveda, Unani, and Siddha. All therapeutic categories are widely used by broad sections of the population. Therefore, in this context, a country like India should take advantage of the potential of traditional medicine systems such as Ayurveda within the framework of an integrated approach.

Research and treatment strategies for Covid-19 are focused on agents that attack or prevent the virus. This puts off the host's consideration which is one of the most important factors in disease dynamics.

This is where we see the glory of Ayurveda. Charaka Samhita, Classic literature of Ayurveda explains epidemics and its etiological factor in Janapadodhwamsa Adhyaya of 
Vimansthana ${ }^{[2]}$. Ayurveda defines immunity in many ways, one of which is the body's ability to prevent disease and prevent its progression.

Ayurveda focuses on strengthening the host components by using classical Ayurvedic formulations and this is how Ayurveda helps to achieve immunity against Covid -19.

Even though, Ayurveda has ample potential and possibilities for both the prevention and treatment of Covid-19, Ayurvedic doctors were not allowed to treat patients suffering from Covid-19. But later, then permission was granted

The guidelines of the Ministry of Health and Family Welfare (MoHFW), states that Ayurveda and Yoga can play a pivotal role in augmenting preventive measures in the management of COVID-19. Also, the current understanding of COVID19 indicates that good immune status is vital for prevention and safeguarding from disease progression. Ayurveda protocol for managing mild COVID-19 has been developed and approved by the Ministry of Ayush.

According to the order issued by the State Department of AYUSH, patients who have tested positive for COVID-19, who are either asymptomatic or have a mild or uncomplicated disease (grouped as Category A patients) may be administered the Ayurveda strategies for the mitigation and rehabilitation of COVID-19 ${ }^{[3]}$. In the light of that order, Ayurveda practitioners have been prescribing medicines to the Covid-19 patients.

So far, no studies have been reported from Kerala regarding the effectiveness of the Ayurveda Treatment approaches. Hence the School of Fundamental Research in Ayurveda designed an observational study to evaluate the impact of the Ayurveda Treatment approaches in the management of Covid -19. Along with understanding how effective Ayurveda is for Covid treatment, the effective formulations identified through the survey will be studied extensively to understand their action and that is the main aim of this observational study.

\section{MATERIALS AND METHODS}

The study was designed as an Observational one and the survey conducted was using a semi-structured questionnaire. The questionnaire with 17 questions was prepared in google forms and circulated among the Ayurveda practitioners in Kerala through WhatsApp and Email. The data is analyzed and presented with appropriate graphs and diagrams.

\section{RESULT}

For the survey, a total of 140 doctors responded, mostly government physicians. The number of Covid patients treated by these 140 practitioners varies from 100 patients to more than 1000 patients. So the study presented here is the report of the assessment of treatment done in not less than 23,470 patients.

The questionnaire generated and the response received is analyzed and data thus obtained is presented using appropriate diagrams. Each question and their responses are detailed below.

\section{Analysis of the survey}

How many Covid-19 positive patients have you treated?

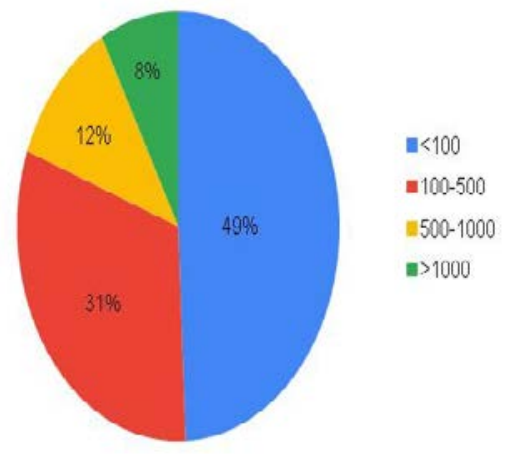

Figure 1: Do you have any experience in treating Covid-19 patients?

Observation: The survey was conducted among the doctors who have got a chance to treat Covid-19 patients. According to the guidelines of the Kerala State Covid Response cell, only ISM doctors were 
allowed to treat the Covid-19 Patients. Hence the majority response received was from ISM doctors. Here we can see, $96 \%$ of participants treated Covid-19 Patients.

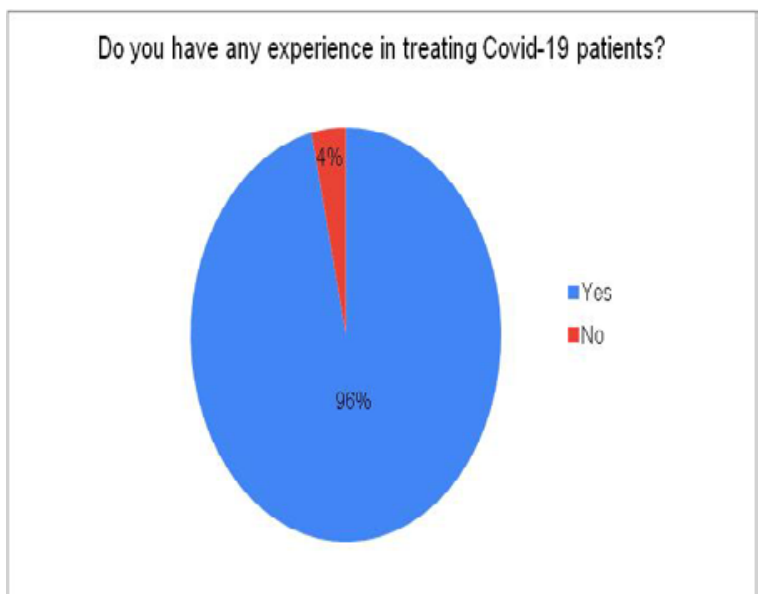

Figure 2: How many Covid-19 positive patients have you treated?

Observation: Of the physicians who treated Covid-19 patients, 51\% treated more than 100 patients, of which $12 \%$ treated more than 500 patients and $8 \%$ treated more than
1000 patients, making a total of not less than 23600 patients.

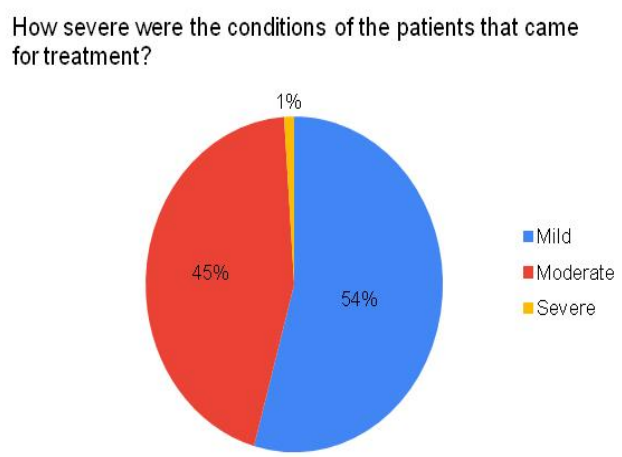

Figure 3: How severe were the conditions of the patients that came for treatment?

Observation: Conditions of $54 \%$ of the patients were mild and $45 \%$ were moderate. Only $1 \%$ of the patients were having severe symptoms. The reason for these figures is the guidelines of the Ministry of AYUSH stating that the Ayurveda Practitioners can treat mild and moderate conditions of Covid-19.

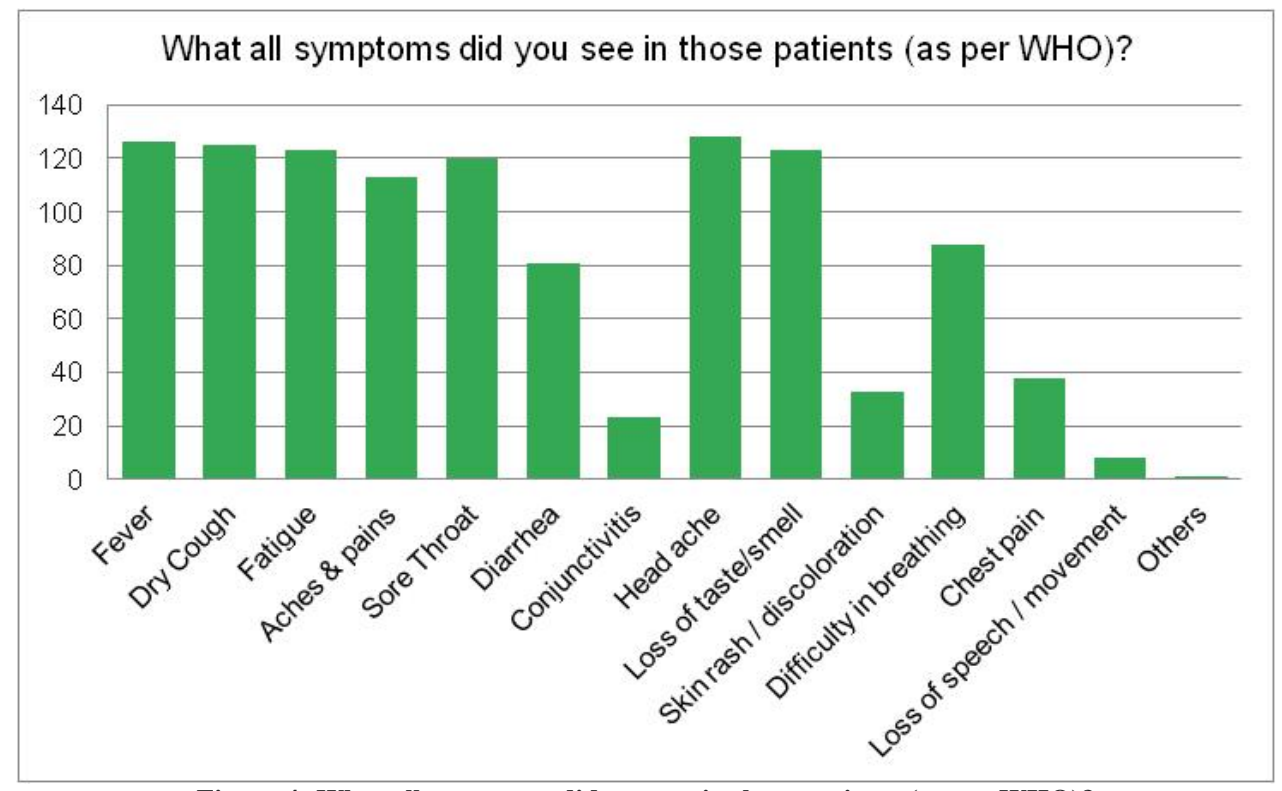

Figure 4: What all symptoms did you see in those patients (as per WHO)?

Observation: The list of symptoms was adopted from the WHO website ${ }^{[4]}$. From the survey, Dry Cough, Fatigue, Headache, loss of taste/smell are the most commonly observed symptoms. More than $90 \%$ of doctors stated fever and headache and 85\% of doctors stated fatigue, sore throat, and dry cough. Less common symptoms like aches \& pain, diarrhoea, conjunctivitis, skin rashes, etc. were also seen in the patients. Serious symptoms were also noticed like Difficulty in breathing by $62.9 \%$, Chest pain $27 \%$, and loss of speech by $8 \%$. 
Have you noticed people becoming Covid positive who have taken Ayurvedic preventive medicine?

Figure 5: Have you noticed people becoming Covid positive who have taken Ayurvedic preventive medicine?

Observation: $69 \%$ of people who have taken Ayurveda Preventive medicine have become Covid positive while $31 \%$ have not.

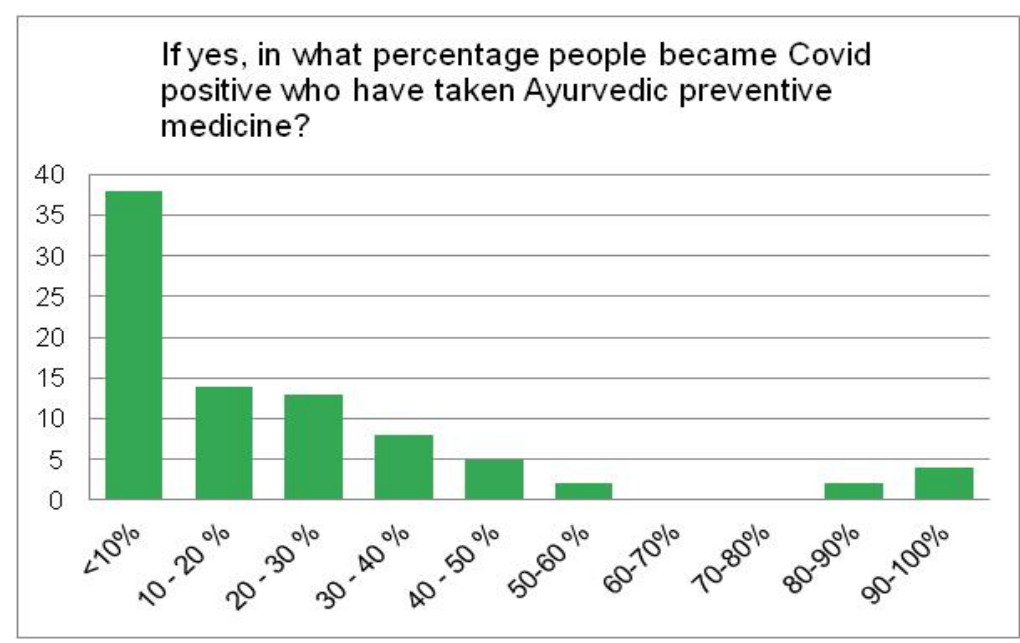

Figure 6: If yes, in what percentage of people became Covid positive who have taken Ayurvedic preventive medicine?

Observation: 39 physicians reported that less than 10\% of their patients got affected with Covid, who have been taken preventive medicines from them while around 8 doctors reported that $50-100 \%$ of patients got affected.

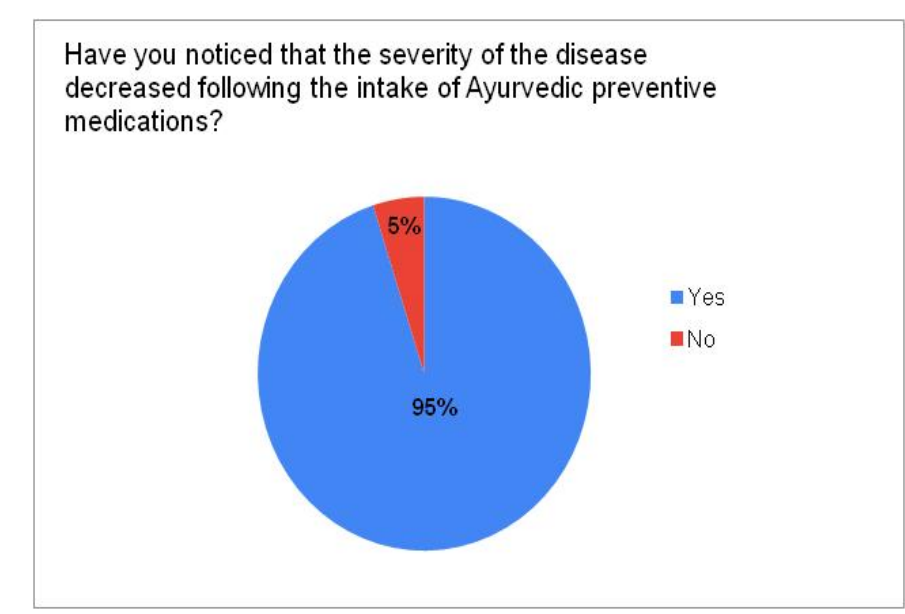

Figure 7: Have you noticed that the severity of the disease decreased following the intake of Ayurvedic preventive medications?

Observation: In 95\% of the patients who are Covid positive, the disease is found less severe. 
Have you noticed that the severity of the disease decreased who have taken Vaccination against Covid$19 ?$

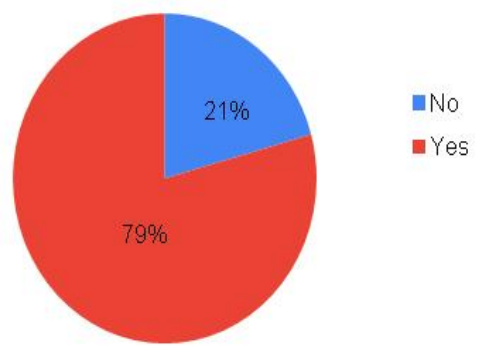

Figure 8: Have you noticed that the severity of the disease decreased who have taken Vaccination against Covid-19?

Observation: $79 \%$ of people who have taken vaccination against Covid-19 have become Covid positive while $21 \%$ have not.

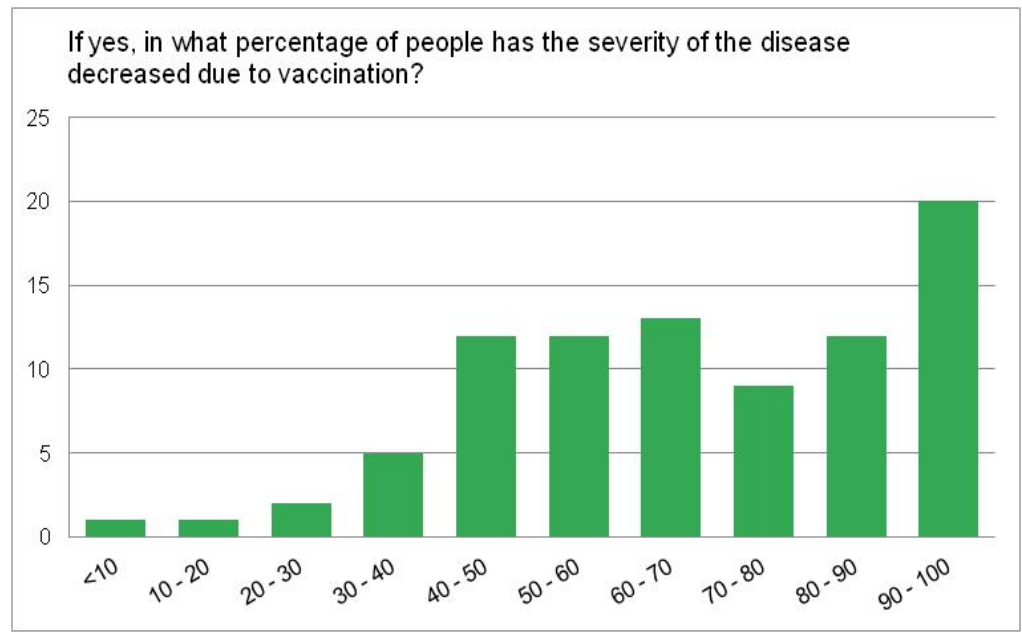

Figure 9: If yes, in what percentage of people have the severity of the disease decreased due to vaccination?

Observation: More than 20 doctors noticed that $90-100 \%$ of patients has the severity of the disease decreased due to vaccination.

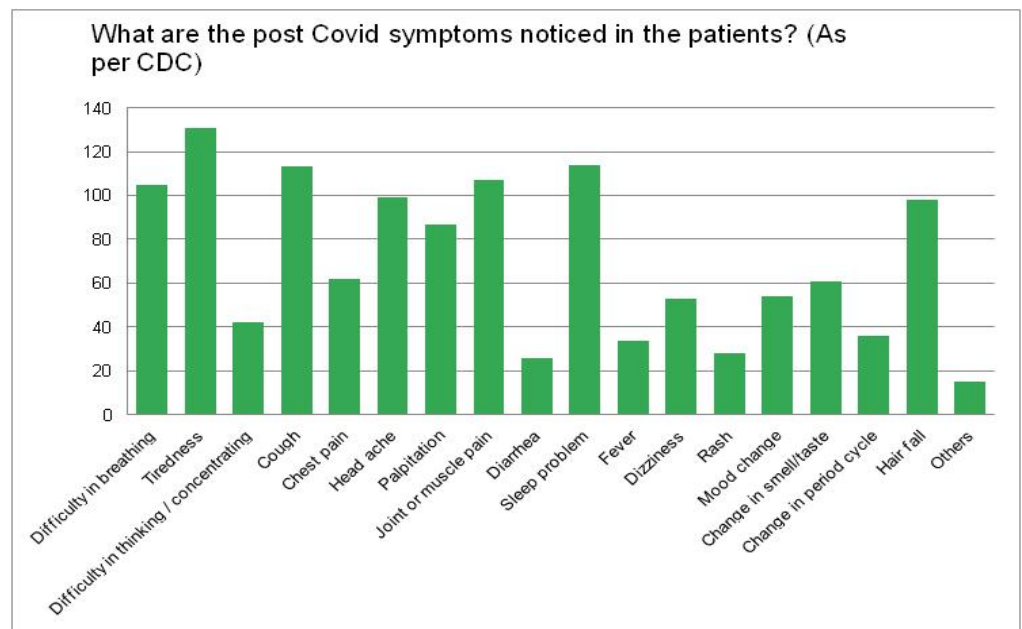

Figure 10: What are the post Covid symptoms noticed in the patients? (As per CDC)

Observation: The list provided here is according to the Centers for Disease Control and Prevention (CDC) ${ }^{[5]}$. According to the survey, the most common post-Covid 
symptoms noticed are Tiredness (93.6\%) followed by sleep problems (81\%), cough (80\%), and joint or muscle pain (76\%). After these, the symptoms that have been reported to be the most common are difficulty in breathing, Chest pain, and Hair fall. Other than the symptoms mentioned by CDC, there were some others noted like Anxiety and fear, blurred vision, watery eyes, anorexia, vision problems, and Gastric issues.

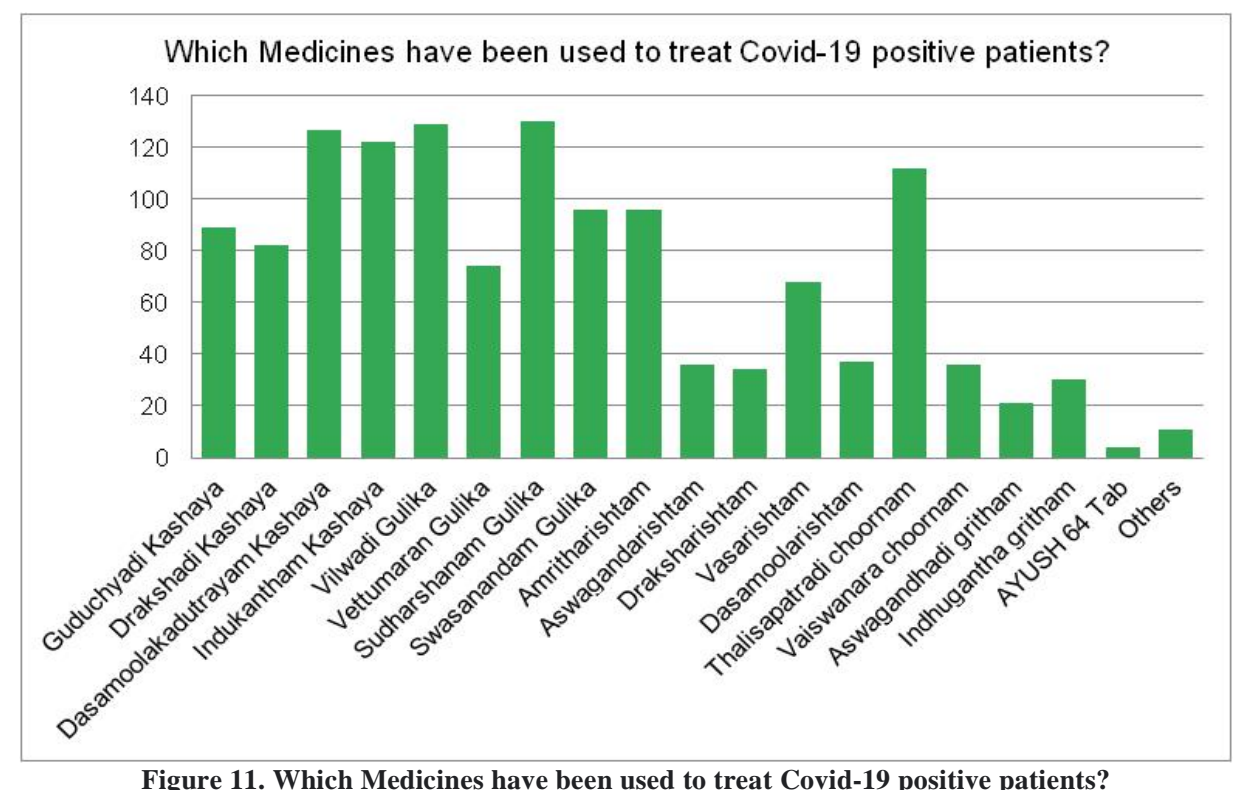

Observation: The most commonly used kashayas identified from this survey are Dasamulakatutrayam Kashayam (90\%) and Indukantham Kashayam (87\%). Among gulikas, Sudarshanam Gulika (92\%), Vilwadi Gulika (92\%) stands top in the list.
Other medications widely used include Thalisapatradi chooram (80\%), Swasanantham Gulika (68\%), Amritharishtam (68\%), and Guduchyadi Kashayam (63\%).

Have you noticed any symptoms that could not be managed effectively with Ayurvedic medicine?

Figure 12: Have you noticed any symptoms that could not be managed effectively with Ayurvedic medicine?

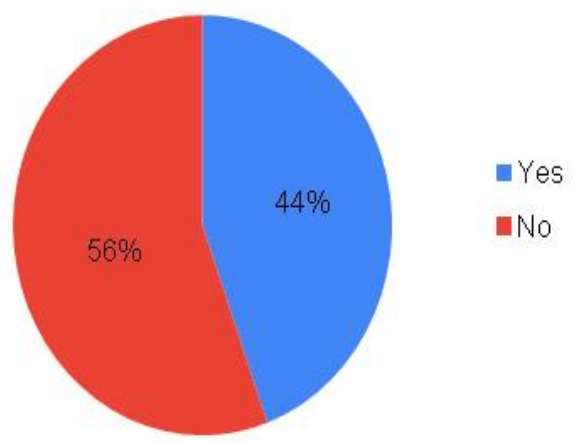

Observation: $56 \%$ of the cases could be treated effectively with Ayurvedic Medicines while $44 \%$ of cases faced some difficulty. 


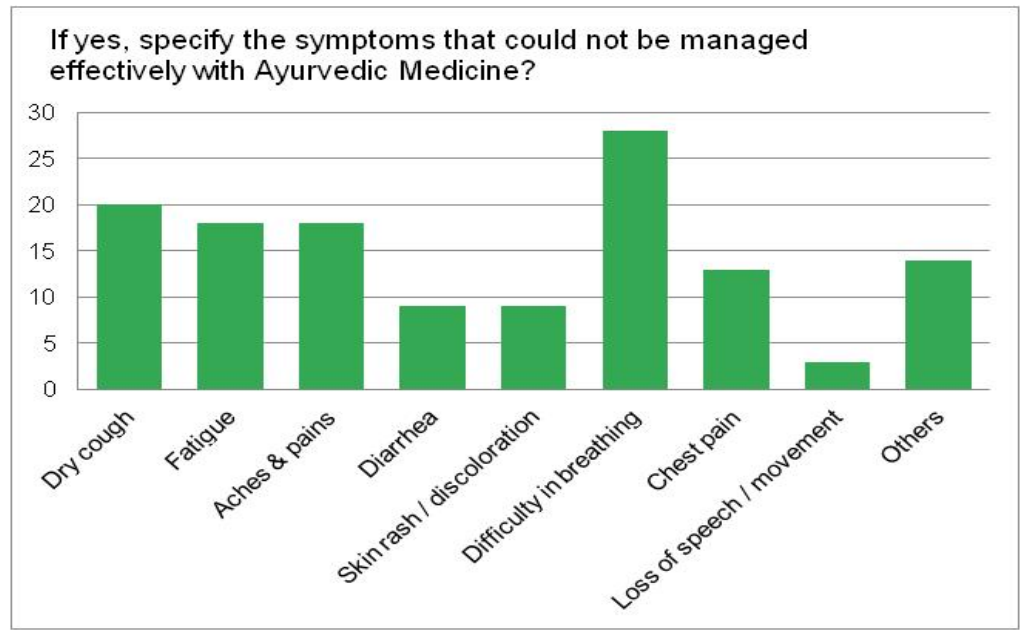

Figure 13: If yes, specify the symptoms that could not be managed effectively with Ayurvedic Medicine?

Observation: The symptoms that could not be managed effectively were Difficulty in Breathing (50\%), Dry Cough (35\%) followed by Fatigue and Aches pain (32\%).

How many days did it take for patients to become Covid negative with the medication you prescribed?

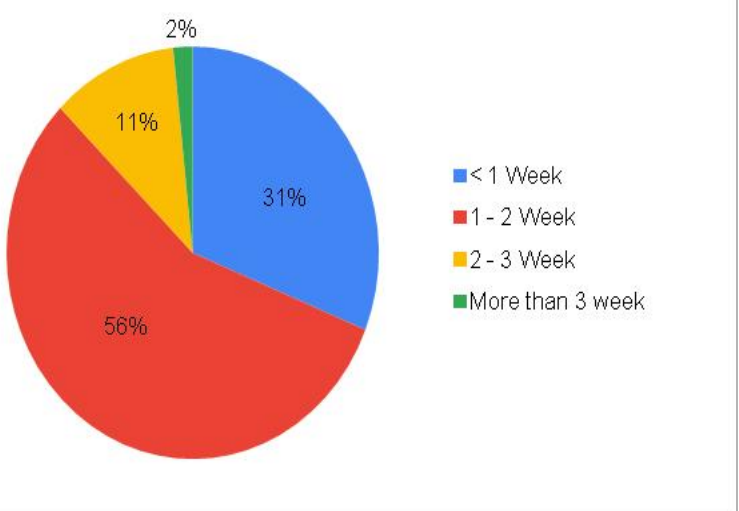

Figure 14: How many days did it take for patients to become Covid negative with the medication you prescribed?

Observation: $56 \%$ of the patients became Covid negative by $1-2$ weeks and $31 \%$ with less than 1 week while $11 \%$ took 2-3 weeks. Only $2 \%$ took more than 3 weeks to become negative.

Have you noticed post-Covid symptoms in patients who are Covid negative by your treatment?

Figure 15: Have you noticed post-Covid symptoms in patients who are Covid negative by your treatment? 
Observation: $19 \%$ of doctors reported that patients who were negative with their treatment had no post-Covid symptoms, but 23\% said the opposite. 58\% said they had both kinds in their experience.

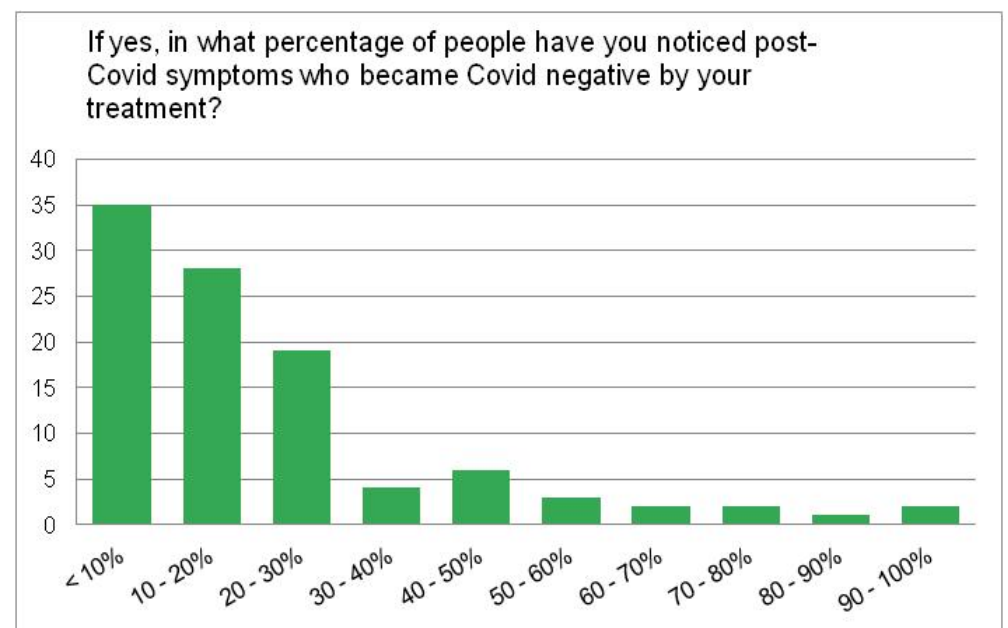

Figure 16: If yes, in what percentage of people have you noticed post-Covid symptoms who became Covid negative by your treatment?

Observation: $35 \%$ of doctors reported that only less than $10 \%$ of their patients who became Covid negative by their treatment had post-Covid symptoms

\section{DISCUSSION}

Kerala state has one of the finest Ayurveda systems of medicine in the country. It has successfully employed the Ayurveda potential both for the prevention and an adjunct treatment option for COVID19.

Unfortunately, Ayurvedic doctors were not authorized to treat the Covid-19 Patients when the pandemic broke out. Therefore, the benefit of Ayurvedic treatment could not reach the public in the beginning. Later, the Ministry of AYUSH gave permission, and still, only ISM doctors were allowed to treat Covid-19 patients, that too only mild and moderate cases. Therefore, almost all the responses received from the survey were from the ISM doctors. Mainly Government Ayurveda dispensaries were prescribing Ayurveda medicines for Covid-19 under various Government schemes like amrutham, bheshajam, punarjani, swasthyam and sukhayushyam. All medications were administered on an OP basis, and IP treatment was not offered.
Data on 23,470 patients receiving Ayurvedic treatment were obtained from this survey with limited feedback from 140 practitioners. These figures show that despite all of the restrictions, the Ayurvedic health care system has been working well since it received approval for treatment.

Globally, the Covid-19 pandemic has challenged health care systems in unprecedented ways. The World Health Organisation (WHO) is the ultimate decision-making authority in matters of health care worldwide and published the COVID-19 Strategic Preparedness and Response Plan, and have published the Cardinal symptoms of Covid-19 as Fever, dry cough, fatigue, headache, and loss of taste or smell. These symptoms were included in the survey questionnaire and all the symptoms mentioned by WHO were noticed by the doctors. Apart from the listed symptoms, others like Lack of appetite, Hair fall, Sleeplessness, short span memory loss, Vomiting, Numbness, Anxiety, fear, constipation, bloating of stomach, flatulence, mood variations, depression, Menstrual problems, Hiccups, etc. were also mentioned.

Ayurveda was allowed to enter the Covid treatment Scenario with the permission to offer preventive medicines 
only. No intervention with active cases was allowed. During that time, the safety measures and the 'Break the chain' campaign were well executed. Ayurveda describes many preventive medicine practices that all have proven beneficial in various ways. Even though these Ayurvedic preventive measures are highly effective, the ability to resist shall depend on the degree of exposure.

If it has been a while since the Ayurvedic preventive medicines have been taken and the factors like exposure are widespread, the antibodies produced after taking the Ayurvedic medicines alone may not be enough to prevent the disease. That must be why those who received preventive medicines also developed the disease. But it must have been able to reduce the severity of the disease.

It is the same with Vaccination against Covid-19. When the vaccination started, safety protocols were strictly followed. Hence exposure was reduced and thereby got good results initially and then it declined along with the fallout of the safety protocols.

COVID-19 crisis contributes to high levels of psychological distress and negatively affects mental health. Fear of disease, being in isolation, and staying away from home will be the major causes of stress and related issues like sleep problems and mental issues. The culture and way of life of Kerala are such that the family is important and hence the fear and isolation following Covid-Positive creates anxiety and makes him insomniac and cause other mental disturbances. Ayurvedic medicines try to make a person completely healthy by incorporating not only physical factors but also mental elements.

In Ayurveda, treatment is determined after understanding the dosha from the symptoms and also considering the Prakriti of the patient. Every disease can be understood and managed based upon its impact on doshas derived from the type of symptoms and areas affected. And hence the list of medicines chosen for treatment is so extensive, as we can see from the survey result. Each Covid Patient may have presented symptoms differently and with every combination of symptoms, the medicine prescribed changes accordingly. That is the greatness and beauty of Ayurvedic treatment.

\section{CONCLUSION}

Ayurveda has ample potential for the prevention and treatment of Covid-19. Based on the type of symptoms and the areas affected, every disease can be understood and managed based on its impact on doshas. The purpose of this survey was to gain an overview of Ayurvedic interventions, both therapeutic and preventive. 140 doctors responded to this who were mostly government medical officers. The study presented here is the report of the assessment of treatment done by these doctors in not less than 23,470 patients.

This survey report affirms that the Ayurvedic treatment method is very effective against Covid-19. It should be noted that the role of Ayurveda in addressing the Covid-19 challenge is invaluable and should be seen as an extension of health care services and support for the bio-medical system. The study of this topic offers a unique opportunity for the extensive study to create scientific evidence.

\section{ACKNOWLEDGEMENT}

We wholeheartedly acknowledge the continuous support and encouragement given by the Hon. Vice-Chancellor, Prof (Dr.) Mohanan Kunnummal, ProViceChancellor, Dr. C P Vijayan, and Registrar, Dr. A K Manoj Kumar of Kerala University of Health Sciences for this study and also for all the endeavours taken up by the School of Fundamental Research in Ayurveda.

\section{Conflict of Interest: None}

\section{Source of Funding: None}


Ethical Approval: Approved

\section{REFERENCES}

1. Corona virus [homepage on the Internet]. (C)2021 WHO [cited 2021 Aug 8]. Available from: $\quad$ https://www.who.int/healthtopics/coronavirus\#tab=tab_1

2. Priyavrat sharma. Charaka Samhitha. (9 ed.). Varanasi: Chowkamba Orientalia; 2004. Vimana sthana. p314

3. Corona virus [homepage on the Internet]. (C)2021 Ministry of Ayush. Government of India. [cited 2021 Aug 8]. Available from: https://www.ayush.gov.in/docs/ayushProtocol-covid-19.pdf

4. Corona virus [homepage on the Internet]. c 2021 WHO [cited 2021 Aug 8]. Available from: https://www.who.int/healthtopics/coronavirus\#tab=tab_3

5. Covid

https://www.cdc.gov/coronavirus/2019ncov/long-term-effects/index.html

How to cite this article: Sailekha P., Sudhikumar $\mathrm{K}$ B. Impact of the ayurveda treatment protocol in the management of COVID -19. Int J Health Sci Res. 2022; 12(1): 36-45. DOI: https://doi. org/10.52403/ijhsr.20220106 